Universidad de Lima

Facultad de Comunicaciones

Carrera de Comunicaciones

\title{
"ANÁLISIS DE LOS PROCESOS EN LA COMUNICACIÓN CORPORATIVA DE LOS CLUBES PROFESIONALES DE LIMA: ESTUDIO EXPLORATORIO"
}

Trabajo de investigación para optar la licenciatura en Comunicación

Pierre Dereck Peláez Alegría

Código 20070821

Asesor

Julio Hevia Garrido-Lecca

Lima - Perú

Julio de 2016 


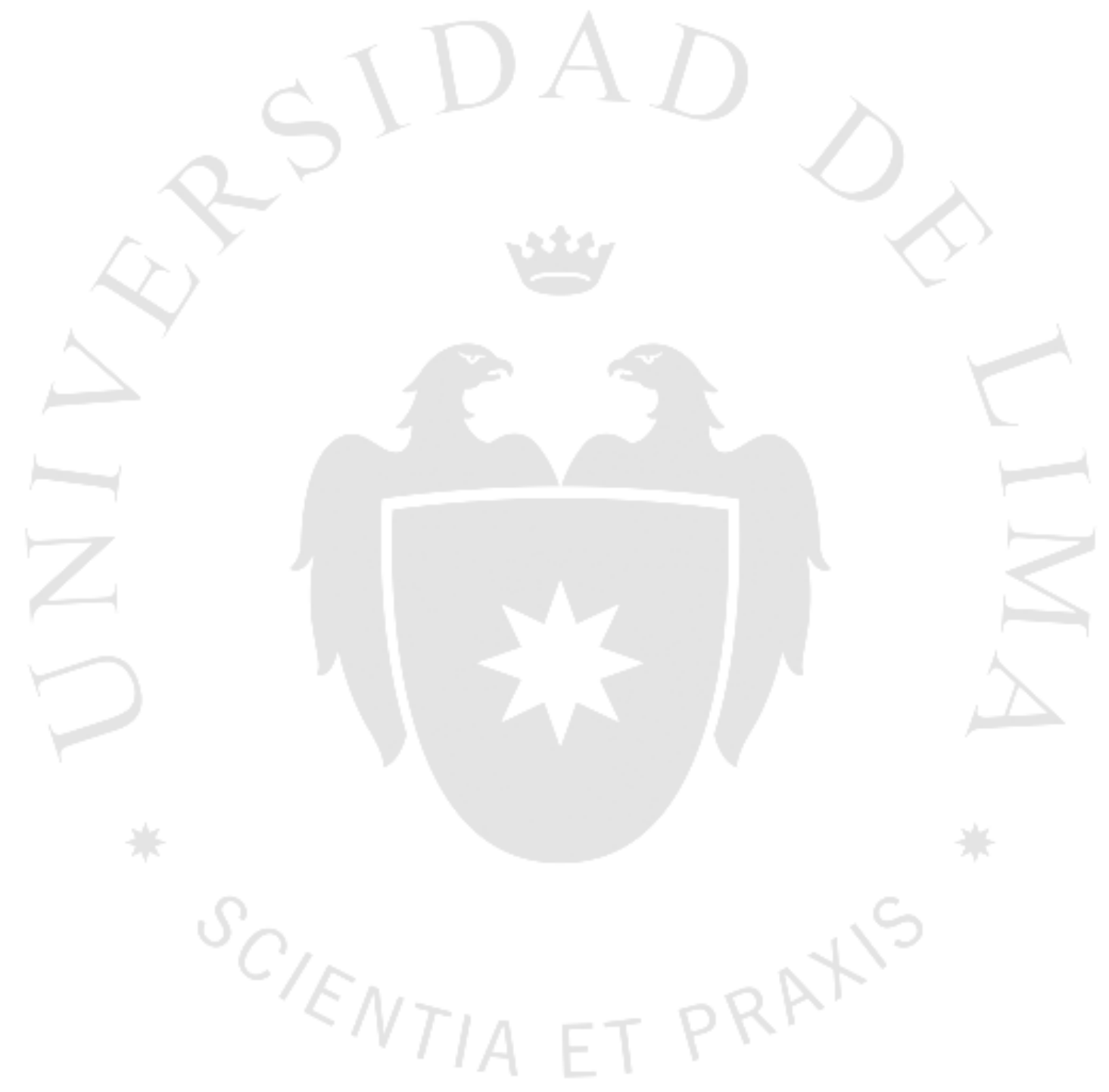




\section{Índice}

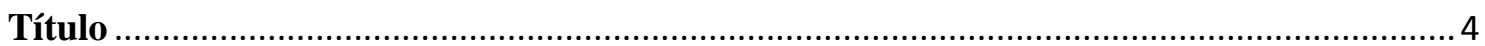

Resumen

Palabras Clave

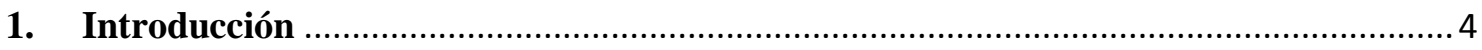

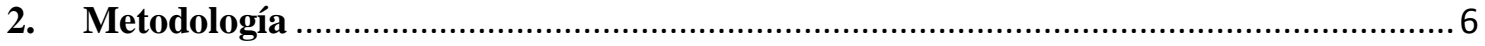

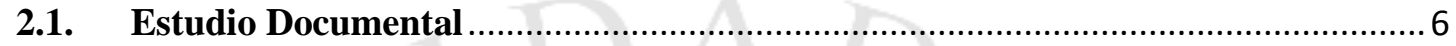

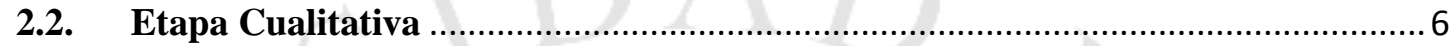

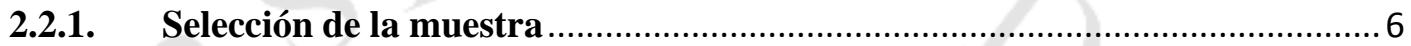

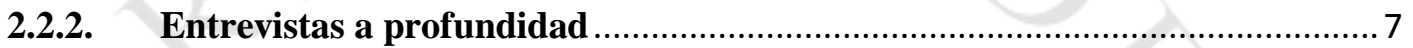

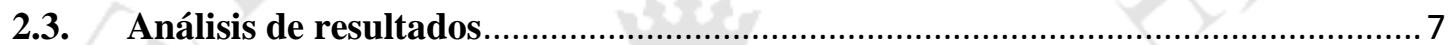

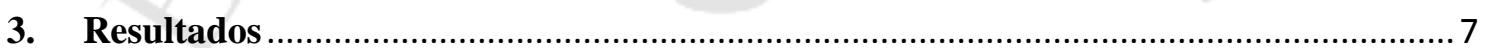

3.1. Club Deportivo Universidad San Martín................................................................. 7

3.1.1. Jefatura de Prensa y Relaciones Públicas ...................................................... 7

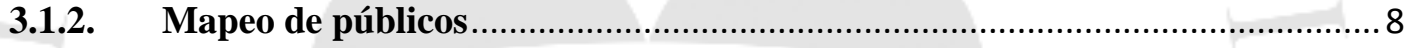

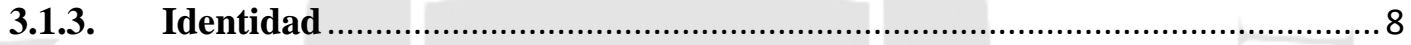

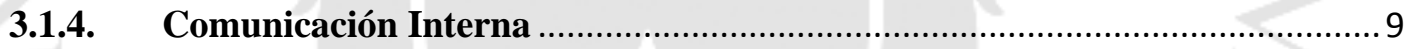

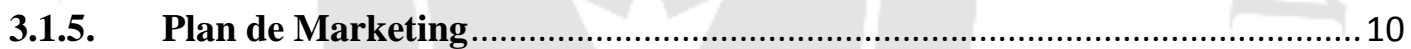

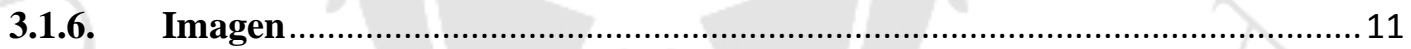

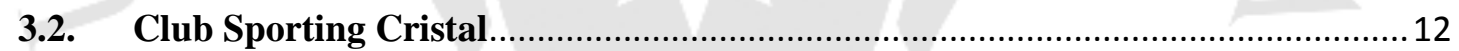

3.2.1. Comité de Comunicación.................................................................................... 12

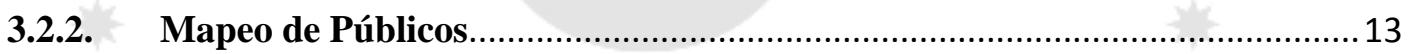

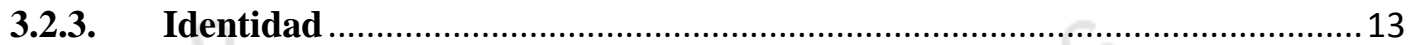

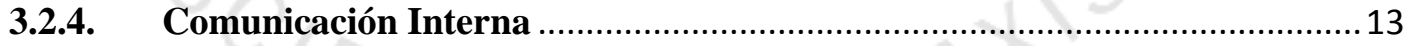

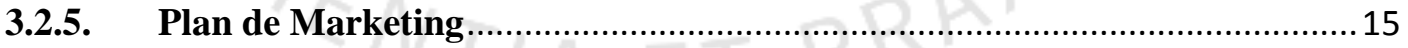

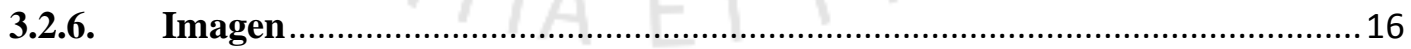

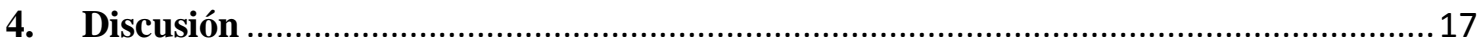

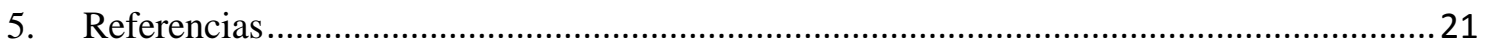




\section{Título}

Análisis de los procesos en la comunicación corporativa de los clubes profesionales de Lima: Estudio exploratorio.

\section{Resumen}

El fútbol peruano atraviesa una crisis institucional, económica y deportiva a consecuencia de la informalidad con la que han sido gestionados los clubes deportivos locales. Sin embargo, no se necesita ir muy lejos para comprobar que las entidades deportivas, gestionadas adecuadamente, y adaptando herramientas del 'management' a sus modelos de negocio, entre los que destaca la comunicación corporativa, han logrado convertirse en instituciones sólidas, reputadas y exitosas.

En ese sentido, resulta preciso advertir cuál es el valor que se le da al 'corporate' en dos de los clubes de fútbol más sólidos del campeonato local (Sporting Cristal y la Universidad San Martín de Porres); del mismo modo, es oportuno conocer la forma en la que se viene desarrollando la comunicación en ambas instituciones; lo cual permitirá realizar una primera aproximación al estado de esta importante herramienta del 'management' en las entidades deportivas nacionales.

\section{Palabras Clave}

Comunicación corporativa, Identidad, Imagen, Relaciones públicas, Marketing deportivo, Gestión deportiva, Fútbol.

\section{Introducción}

El contexto en el que se desarrolla el fútbol de nuestros días es distinto al del siglo pasado: El número de aficionados asciende año tras año, la relevancia que adquiere el mismo en los medios masivos es mayor, las empresas destinan hoy en día más porcentaje de su presupuesto de comunicación al patrocinio deportivo, los ingresos de los clubes europeos han ascendido significativamente, etc. ¿Cuál es la razón?

La gestión de las principales entidades deportivas del mundo se profesionalizó desde hace unas décadas. El desarrollo de la industria del deporte es una realidad en países del viejo continente, donde los clubes lograron - en muchos casos - generar 
réditos mediante una excelente gestión de sus marcas y comercializando el máximo de su actividad deportiva. En la actualidad, éstos se han convertido en verdaderas multinacionales de entretenimiento, proveedoras de un espectáculo mundial del más alto nivel (Soriano, 2013).

La comunicación corporativa resultó fundamental en esta conversión del fútbol en un producto apto para el consumo de audiencias globales, proceso denominado 'comodificación' (López, 2012). Numerosas entidades deportivas emplearon estrategias de comunicación a fin de desarrollar una esencia de marca, una identidad capaz de diferenciarlas del resto, permitiendo generar conexiones más profundas con sus seguidores y convertirlas en íconos de alto valor comercial (Varela \& Franch, 2014).

En Latinoamérica, la pasión por el fútbol se vive con mayor intensidad. El vínculo que une a los fanáticos con sus equipos favoritos destaca a nivel mundial por su colorido, fervor, y pasión. De acuerdo a Rinke (2007), el fútbol es - en América Latina aún más que en Europa - una fuente de identidad a nivel regional, nacional y continental; así como una fuente de inspiración para la producción artística y literaria. Indudablemente, este escenario representa una oportunidad para el florecimiento de la industria del deporte en los países de la región.

Los clubes más relevantes de Brasil, Argentina, México, entre otros, se enfrentaron al reto de convertir a sus fans en clientes a través de la monetización (Varela \& Franch, 2014). Al igual que sus similares europeos, utilizaron herramientas de comunicación y marketing de manera eficiente logrando salvaguardar su filosofía, su identidad, y dirigir sus actividades comerciales, diversificando sus fuentes de ingresos e incrementando sus ganancias considerablemente.

Sin embargo, los clubes de fútbol peruanos han sido incapaces de adaptarse al nuevo paradigma empresarial; en el cual, la identidad, la cultura, la acción, la comunicación y la imagen se han convertido en los vectores que rigen la gestión estratégica de las organizaciones (Costa, 2003). Por el contrario, estarían comunicándose todavía con sus grupos de interés mediante un modelo asimétricounidireccional, también llamado "de agencia de prensa, en el cual las organizaciones buscan solamente influir en la opinión pública, sin interesarse realmente por sus públicos" (Di Genova, 2012, pág. 13). 
He ahí el interés de esta investigación: Conocer los procesos de comunicación llevados a cabo en dos de los clubes de fútbol profesional que sobresalen por una administración ejemplar - como son el Club Sporting Cristal y el Club Deportivo Universidad de San Martín - a fin de detectar las debilidades de los mismos en términos de comunicación y marketing, y dar a conocer los desafíos que enfrentan los directivos responsables.

\section{Metodología}

La metodología a implementar en el presente trabajo de investigación sigue el modelo empleado por Guillermo Sanahuja Peris (2012) en la publicación Análisis de los procesos en la comunicación corporativa de los clubes deportivos profesionales españoles. Estudio Exploratorio. En ese sentido, la presente investigación constará de 3 etapas: Estudio Documental, Etapa Cualitativa, Etapa Contraste y Análisis de Resultados.

\subsection{Estudio Documental}

Esta etapa corresponde a la exploración de las principales aportaciones teóricas de la comunicación corporativa, marketing deportivo y de las técnicas de dirección y gestión de empresas aplicadas a la industria del fútbol. Además, revisión de los lineamientos que siguen, en términos de comunicación, clubes exitosos en el resto del mundo. Todo ello permitirá preparar las herramientas de investigación a emplear en la siguiente etapa.

\subsection{Etapa Cualitativa}

\subsubsection{Selección de la muestra}

Los clubes deportivos que participan de la primera división del fútbol profesional suman 17 en total. Los representantes del interior del país sumaron 12 durante el año 2015; mientras que los de la capital, 05 clubes.

Cabe destacar, sólo 03 instituciones del total ejercen funciones bajo la denominación de empresas privadas con fines de lucro: Sporting Cristal (Lima), 
Club Deportivo Universidad San Martín de Porres (Lima) y el Club Deportivo Universidad César Vallejo (La Libertad).

Dado que el presente trabajo corresponde a una primera exploración sobre la gestión de la comunicación corporativa en los clubes de fútbol profesional, se ha tomado la decisión de considerar a 02 de las 05 instituciones capitalinas: Club Deportivo Universidad San Martín de Porres, y el Club Sporting Cristal. Ambas son reconocidas - según prensa especializada - por ser instituciones que han llevado a cabo una gestión responsable desde hace varios años, tanto en lo deportivo como en lo administrativo; por ende, consideradas por los otros clubes como entidades referentes en temas de gestión deportiva.

\subsubsection{Entrevistas a profundidad}

Se desarrollarán entrevistas a profundidad a los responsables de la comunicación/marketing en cada uno de las instituciones. En el Club Universidad San Martín, la responsabilidad absoluta recae sobre Ricardo Huaytalla, Jefe de Prensa y Relaciones Públicas. Por otro lado, en el Sporting Cristal, se realizarán dos entrevistas: La primera al Jefe de Comunicaciones Internas y Externas, Luis Oyola; la segunda, a Noé Miranda, responsable del Área Comercial y Marketing del club.

\subsection{Análisis de resultados}

Sobre los resultados obtenidos producto de las entrevistas a profundidad desarrolladas, se podrá evaluar los conceptos manejados por el personal responsable, y las tareas realizadas por ellos referentes a la gestión de intangibles y desarrollo comercial.

\section{Resultados}

\subsection{Club Deportivo Universidad San Martín}

\subsubsection{Jefatura de Prensa y Relaciones Públicas}

Ricardo Huaytalla, Jefe de Prensa y R.R. P.P. del Club Universidad de San Martín de Porres, labora en el club desde hace 08 años. Es comunicador, graduado de la USMP, sin estudios de postgrado. 
El área que dirige está conformada por 04 personas, de las cuales, dos tienen la responsabilidad de gestionar la relación con los medios de prensa e 'hinchas' del club. Es decir, elaborar las notas de prensa dirigidas a los medios deportivos especializados, coordinar el acceso a los periodistas durante la semana, elaborar los comunicados internos y gestionar las redes sociales.

Las otras dos personas dedican la mayor parte de su tiempo a la edición de videos a solicitud del Director Técnico de fútbol, el argentino Christian Díaz. Este material audiovisual contiene pasajes de los partidos que le servirán al entrenador para transmitir su idea de juego a los jugadores.

\subsubsection{Mapeo de públicos}

Según Huaytalla, destacan los siguientes: Los medios de prensa; con los hinchas (mayormente a través de redes sociales); jugadores e integrantes del cuerpo técnico; los niños, adolescentes y jóvenes que componen las divisiones menores del club; los niños participantes de las escuelas de fútbol administradas por la institución; y las tres personas adicionales que conforman el personal administrativo.

Nótese, no se mapean empresas con el potencial de convertirse en sponsors del club. Se reconocen una vez que éstas se acercan a la institución solicitando información sobre el coste de los 'espacios disponibles' en la camiseta. Es el caso de una empresa de apuestas deportivas - cuyo nombre se mantiene en reserva - interesada en auspiciar al club 'santo' el próximo año, en caso logre mantenerse en primera división.

\subsubsection{Identidad}

El concepto de identidad es manejado someramente por el responsable del área; en consecuencia, no existe una estrategia destinada a expresarla de manera sostenida.

Según Huaytalla, el primer equipo representa de forma acertada aquello que identifica a la institución: seriedad en la manera de trabajar, cero escándalos y juego limpio. Esta manera de hacer las cosas es comunicada personalmente a todo nuevo jugador una vez confirmado su ingreso a la plantilla del club; lo mismo ocurre con todos los miembros de las divisiones menores. Sin embargo, no existe una estrategia formal, dirigida por especialistas, destinada a expresar la filosofía del club al 
personal administrativo, ni a reforzar la misma a los deportistas del club durante el transcurso del año.

La infraestructura del club santo no es utilizada en lo absoluto para transmitir la identidad de la institución. Salvo por la colocación de paneles publicitarios en donde puede observarse los patrocinadores del equipo, nada da señales de pertenecer a una entidad en particular.

\section{Misión.-}

"Buscar fomentar la práctica profesional del fútbol, con fundamentos sólidos y valores éticos, para lograr así el desarrollo del fútbol peruano”.

\section{Visión.-}

"Posicionarnos como el mejor equipo de fútbol del país, a través de la formación de los mejores jugadores, para lograr consolidarnos como un equipo íntegro, que se caracterice por la constancia, esfuerzo y calidad en el desempeño de su trabajo, logrando así la obtención de sus objetivos".

\section{Valores.-}

Disciplina, Responsabilidad, Orden, Honor, Actitud.

\subsubsection{Comunicación Interna}

No existe un plan de comunicación interna puesto que no se le considera necesaria debido el número de empleados que componen en público interno (60). En el mayor de los casos, el club transmite información mas no dialoga con sus colaboradores.

La comunicación en cascada es la herramienta utilizada por la Jefatura de Prensa y Relaciones Públicas al momento de transmitir alguna información a un público interno determinado. No obstante, no existen lineamientos sobre los aspectos que determinen su utilización.

Los jefes de equipo, directores técnicos y personal administrativo de alto rango reciben la información directamente desde la dirigencia del club, siendo ellos quienes luego deberán transmitirla a sus subordinados. Las sugerencias, comentarios, solicitudes, etc., propias de cualquier jugador o colaborador 
administrativo, deberán ser dirigidas al superior inmediato, quien, a su vez, la trasladará al ente respectivo. La situación se repite en los demás equipos, tanto de reservas, como los de divisiones inferiores.

Las plataformas de comunicación más utilizadas son los comunicados escritos, correos virtuales, de manera presencial en reuniones mensuales, y murales, estos últimos utilizados mayormente para recordar fechas importantes e información útil relevante a recordar (Hora de entrenamientos, días de descanso, cumpleaños, etc.).

\subsubsection{Plan de Marketing}

El desarrollo de un plan de marketing está supeditado a los resultados deportivos. Es decir, "si el club obtiene buenos resultados, podemos presentar ideas que pueden ser aprobadas por directorio; pero, si los resultados no se dan, entonces todo ello se limita".

El desempeño del primer equipo en el campeonato peruano la temporada 2015 fue bastante irregular. Si bien obtuvo el segundo lugar en el primer torneo disputado del año, no logró sostener el buen rendimiento demostrado. Su performance decayó sumando varios partidos sin poder ganar, resultados deportivos que llevaron al club a la zona de descenso durante gran parte del año y obligándolo a luchar su permanencia en primera división.

Por tanto, toda estrategia, táctica y acción de marketing orientada a la captación de nuevas empresas patrocinadoras - principal ingreso del club además de la subvención de la universidad - fue dejada de lado.

La institución cuenta con tres sponsors oficiales: Umbro (marca deportiva), Herbalife (marca de vitamínicos), y Cosapi Data (tecnología de información). Sin embargo, lo único que se les ofrece es presencia en la camiseta, en banners durante conferencias de prensas, banners en sede deportiva y en el estadio durante los partidos. La potencia de los beneficios otorgados a las marcas dependerá también de los resultados deportivos: El equipo carece de una fanaticada numerosa, por tanto, los medios escritos sólo le otorgan media cara interior, como máximo. Si el equipo se ubica en las primeras posiciones, tendrá la posibilidad de aparecer en portada (camiseta incluida), elevar el número de medios que asistan a las conferencias de prensa y cubran los entrenamientos. 
Por otro lado, el club es consciente de su limitado número de aficionados; sin embargo, no desarrolla actividades orientadas a incrementar el número de aficionados. "Los hinchas llegarán solos, con los resultados, es un trabajo a largo plazo", afirmó.

Tampoco se llevan a cabo acciones orientadas a desarrollar actividades comerciales capaces de 'monetizar' la afición de sus seguidores. “Así como están las cosas, no puedo ir donde los dirigentes a presentar ideas. Teníamos en mente vender merchandising, pero no es el momento. Los resultados deportivos no avalan el desarrollo de los mismos", comentó Huaytalla.

\subsubsection{Imagen}

La imagen corporativa, entendida como la forma en la que los públicos perciben a la institución, se sostiene sobre la seriedad en el tema de cumplimiento de contratos. No se realizan actividades que permitan mostrarle al público externo la identidad del club. Ello obedece al escaso reforzamiento de la identidad en el primer equipo de fútbol profesional.

Huaytalla manifestó el interés de una empresa de apuestas deportivas interesada en convertirse en sponsor del club el año 2016; no obstante, al preguntársele los motivos que habrían llevado a dicha compañía a interesarse en el club santo, el comunicador respondió, "por la seriedad de la institución". En ese sentido, puede afirmarse que las empresas asocian su imagen a la del club santo sólo impulsados por la 'seriedad' que esta institución transmite mas no por los valores descritos en la filosofía.

Los aficionados e hinchas del club - todavía muy pocos - pueden enterarse de las noticias del equipo a través de redes sociales: Facebook, Twitter. Sin embargo, estos medios de comunicación se limitan a cumplir un rol informativo, no dialogante, dejándole esta labor a páginas autónomas creadas por hinchas del club, en donde se exponen comentarios sin un lineamiento claro.

En general, no se realizan esfuerzos por conocer, medir, ni estudiar la forma en la que el club es percibido realmente por sus grupos de interés puesto que no se considera necesario hacerlo. Pese a lo descrito, la organización es reconocida - 
puede notarse en los comentarios de sus seguidores en redes sociales - por la 'seriedad' en la forma en la que es administrada.

\subsection{Club Sporting Cristal}

\subsubsection{Comité de Comunicación}

El periodista Luis Oyola, licenciado en ciencias de la comunicación por la Universidad San Martín de Porres, dirige la Jefatura de Comunicaciones del Club Sporting Cristal S.A. El área que comanda está conformada por tres personas, incluyéndolo. Junto a él trabaja un 'community manager', responsable de gestionar la comunicación 2.0, y un periodista encargado de elaborar notas de prensa y tomar las fotografías requeridas.

Antes de desempeñar el cargo de Jefe de Comunicaciones en el club celeste, labor que viene ejerciendo desde hace 05 años, Oyola trabajó como Jefe de Informaciones en TV Perú (01 año), Jefe de Prensa de la Federación Peruana de Fútbol (02 años), y previamente, como Editor General de Deportes en el Grupo La República (12 años), siendo el trabajo en prensa la base de su experiencia laboral.

Las funciones que desempeña el área son, principalmente, gestionar la relación con los medios de prensa, proporcionándoles información sobre el acontecer del club y/o los accesos correspondientes para que los periodistas cumplan su trabajo. Asimismo, mantener informados a los hinchas sobre las novedades del club, promociones, resultados, fechas importantes, entre otras noticias.

El área trabaja supervisada por un Comité de Comunicación, conformado por profesionales de Backus - empresa a la que pertenece el club - y dos especialistas de la consultora española Llorente \& Cuenca, quienes se reúnen semanalmente a fin de tomar las decisiones más importantes en materia de gestión de la identidad, imagen, y acciones de marketing. Está presidido por Víctor Baracco (Gerente General del Country Club La Planicie), quien se mantuvo como Gerente Comercial y de Comunicaciones en el club hasta el año 2014, tras un paso como Business Development Manager in Corporate Partnerships en el 'Chicago Fire', club de la Liga de fútbol de Estados Unidos. 
Luis Oyola forma parte de este Comité, junto a Cecilia Quevedo (Coordinadora de Comunicaciones en Backus), Malena Morales (Directora de Desarrollo Sostenible y Reputación Corporativa en Backus y Directora Ejecutiva de la Fundación Backus), Carlos Benavides (Gerente General del Club Sporting Cristal S.A.), así como José Carlos Antón, Raquel Tintaya, y el abogado Johan Schoenfeld, estos tres últimos de la consultora en mención.

Los profesionales que conforman el comité de Comunicación del Club Sporting Cristal provienen de diversas disciplinas: Periodismo, Responsabilidad Social, Comunicación, Gestión deportiva, Marketing, etc. Este aspecto mejora la toma de decisiones referidas a todo aquello que concierne la comunicación corporativa.

\subsubsection{Mapeo de Públicos}

Los stakeholders del club se dividen en internos y externos. Los internos son los directivos, jugadores, los administrativos, y las divisiones menores. Por otro lado, los externos serían los hinchas - divididos categóricamente según su grado de afinidad con el club y asistencia al estadio -, las empresas patrocinadoras y potenciales sponsors.

\subsubsection{Identidad}

La identidad del club se viene trabajando desde el Comité de Comunicación. Integridad, Lealtad, Compromiso, Equilibrio y Unidad son los valores de la institución. El cumplimiento de estos valores es aquello que le dará sentido a la denominada 'Raza Celeste', apelativo con el que intentan diferenciar a todos los miembros de la 'familia rímense' de los demás clubes del Perú y el mundo.

“Ser de la 'Raza Celeste' te describe como una persona aguerrida y un fanático intenso, pero a la vez te define como un profesional con una fuerte ambición por el triunfo y con una actitud obsesiva por conseguirlo de forma correcta y honesta. No eres el mejor, no eres el peor. No eres soberbio y tampoco sumiso. Sencillamente perteneces a una legión de diferentes", se lee en la página web del club.

\subsubsection{Comunicación Interna}

¿Cómo se fomentan los valores? Primero, las 'viejas glorias' del club - ex jugadores inolvidables para todo aficionado del Sporting Cristal e identificados 
totalmente con el club - cumplen un rol fundamental. El Jefe de Comunicaciones, Luis Oyola, comentó, “ellos representan nuestras historia, nuestros logros, así como lo que queremos seguir siendo: Un club grande en todo sentido, un club de valores".

Para él, su sola presencia genera un impacto importante; sin embargo, no basta. Por tanto, los ex jugadores presencian los entrenamientos de las divisiones menores y comparten con ellos experiencias y anécdotas de los años en los que se vestían de corto. Además, resaltan la forma en la que fueron forjando el cariño que le tienen a la institución, y qué es lo que esta representa para ellos.

Segundo, utilizando las instalaciones del club adecuadamente. Las personas que ingresan a la sede institucional de Sporting Cristal, ubicada en la Florida, observarán desde el momento en el que ingresan posters titulados "Raza Celeste", en donde están los rostros de los 'ídolos' del club - de diferentes épocas encerrados en una corona de laurel y sobre una frase alusiva al mismo. Por ejemplo, debajo de la imagen de Alberto Gallardo se lee, "Dicen que llegaba al gol sin escalas".

Del mismo modo, se observa debajo de la imagen de Julio César Uribe, "Dicen que hizo brillar la celeste como un diamante"; de Jorge Soto, "Dicen que nunca se cansó de perseguir la pelota"; debajo de Orlando La Torre, "Dicen que alzaba la ceja y temblaban los arietes"; de Julio César Balerio, "Dicen que fue el viejo y sabio guardián de una poderosa máquina celeste"; de Julinho, "Dicen que sin música los hacía bailar"; de Roberto Palacios, "Se dice que su pecho habla por él”; etc.

"Somos el club que nació campeón, y eso también lo queremos transmitir", comentó Oyola. La ubicación del último trofeo ganado por el club a pocos metros de la entrada, correspondiente a la temporada pasada, evidencia su afán por emitir tal mensaje. Es inevitable ver la Copa Movistar 2014 al ingresar, conservada en una vitrina ubicada en medio de una estructura decorada con fotografías del equipo campeón.

Los interiores de las oficinas administrativas también son aprovechados. El color celeste prima en todas las paredes, imágenes de los equipos campeones están colocadas en diversos ambientes, junto a recortes de periódico que destacan alguna actuación. 
Finalmente, todo el personal administrativo del club viste con camisetas proporcionadas por la marca oficial que viste al club. En todas ellas está presente el escudo de la institución. Sin embargo, más allá de aquel detalle, resalta lo siguiente, "cada uno de los que trabajamos aquí somos hinchas del club, sabemos quiénes somos y hacia donde apuntamos".

¿Cómo asegura que los colaboradores sepan cómo está haciendo su trabajo? ¿Cuán importante es su trabajo? Al igual que el resto de los mensajes, estos se transmiten de forma personal, o vía correo electrónico. "Valoro la relación interpersonal. Nada más valioso que acercarme a cada uno de ellos a decirle, 'gracias, tu trabajo es sumamente valiosos para el equipo y para la institución””, aseguró Oyola.

\subsubsection{Plan de Marketing}

La Gerencia de Marketing del club Sporting Cristal, creada hace dos años, está integrada por cinco personas. Entre ellos, Noé Miranda, administrador graduado de la Universidad San Ignacio del Oyola, hoy responsable de marketing en el club.

Actualmente, Backus financia el 30\% del presupuesto de Sporting Cristal, sin generarle réditos a la corporación. El objetivo de la gerencia de marketing es lograr convertirse en ser una institución auto-sostenible en de los próximos tres años. Para lo cual, se ha desarrollado un plan de marketing con actividades destinadas a lograrlo.

"Víctor Baracco es la persona que impulsó la creación de la gerencia de marketing, la cual trabaja orientada hacia la fidelización de los hinchas, la captación de nuevos seguidores y el crecimiento económico de la institución en base a la innovación, desarrollo y venta de nuevos productos”, afirmó Miranda.

El ingreso más importante es aquel proveniente de los sponsor. ¿Qué le ofrece el club a sus patrocinadores? "Nos consideramos una plataforma ideal para que las empresas generen vínculos con nuestros hinchas y asocien su marca al prestigio del club, prestigio que debe verse tanto dentro como fuera de la cancha”, comentó.

En segundo lugar, se ubica el dinero recaudado por concepto de taquilla. La venta del 'Pase Celeste' ha mejorado la asistencia al estadio los últimos años; este es un abono que permite pagar anticipadamente por una serie de partidos a un coste 
mucho menor al que se pagaría por la compra de tickets semanal. Finalmente, se ubica el ingreso por actividades comerciales, sea merchandising y academias de fútbol de menores.

"La actividad del marketing debe crecer para ser auto-sostenibles, y con ella la venta de más productos afines al club. En el 2016 cumpliremos 60 años y vamos a aprovechar el momento con nuevos productos", manifestó Miranda.

¿Cuál es el gran desafío? El principal producto que ofrece Sporting Cristal es el fútbol, así lo entiende Miranda. En ese sentido, el desafío es lidiar con un público que, de acuerdo a Miranda, es muy voluble e inconstante. "El consumidor de fútbol local es sensible a factores externos, por ejemplo, los resultados de la selección. Si a la selección peruana le va mal, repercute en una menor asistencia al estadio, menor visitas a la web, etc. Lo mismo con los hechos de violencia.”, señala.

En segundo término, es importante luchar contra la poca costumbre del aficionado de consumir productos originales asociados al fútbol. "Es complicado competir en precios. Una camiseta bamba por ejemplo, cuesta 15 soles y la encuentras en la puerta del estadio; una original vale más de 100 soles”, comentó. Además, para Miranda, la periodicidad con la que se consumen estos productos es demasiado larga, "el hincha compra una camiseta original y no la vuelve a comprar hasta después de 2, 4 o 6 años, en otros lados existe la costumbre de comprar merchandising más seguido", concluyó.

\subsubsection{Imagen}

El comité de comunicación dirige el monitoreo de la información que es publicada referente al club. "Trabajamos para que las noticias negativas que atenten contra la imagen del club en los medios no superen el 5\%. Nos hemos mantenido dentro del rango los últimos meses", declaró Oyola.

La violencia generada por las barras bravas es un tema que el club ha decidido enfrentar pues considera afecta su imagen. Sporting Cristal desarrolló el programa "Desarrollando la Raza Celeste", junto a la ONG Creser. "Somos la única institución deportiva que se preocupa por el desarrollo y futuro de sus barristas", resaltó Oyola. 
¿En qué consiste? El proyecto está orientado a promover el desarrollo personal de los barristas fortaleciendo su autoestima y convirtiéndolos en líderes positivos, conseguir el fortalecimiento organizacional de la barra organizada y convertirla en agente comprometida con la sociedad, desarrollar sus capacidades laborales y ayudarles a insertarse en el mercado laboral, y, finalmente, conseguir que los barristas del programa transmitan el mensaje entre sus compañeros. Este programa fue saludado por los medios de prensa y toda la opinión pública cuando comenzó en el 2012.

Por otro lado, con respecto a las redes sociales, no asumen una actitud dialogante con sus aficionados cuando se hace notorio algún comentario negativo en alguna de sus publicaciones. En pocas ocasiones, la voz de protesta del hincha se traslada a las redes sociales, quien reclama algo. No existe al respecto una respuesta oficial; sin embargo, esto es relativo a los resultados deportivos.

\section{Discusión}

La gestión de la comunicación, en ambos clubes, es sumamente diferente. Por un lado, en el Club Deportivo San Martín de Porres (USMP), la principal tarea del responsable de comunicación, Ricardo Huaytalla, consiste en proporcionar información a los medios de prensa especializados. Es decir, labores similares a las que ejercían los relacionistas públicos a inicios del Siglo XX., cuando predominaba el modelo asimétrico-unidireccional de comunicación.

En aquel entonces, las acciones de comunicación ejecutadas por las organizaciones tenían el único objetivo de influir en la opinión pública en beneficio propio. Las Relaciones Públicas (RRPP), “entendidas como el proceso mediante el cual se encontraban y difundían los aspectos de una organización que eran de interés público" (Di Genova, 2012, pág. 13), alcanzaron gran relevancia en el mundo empresarial.

Décadas más tarde, la disciplina descrita se aproximaría al modelo simétricobidireccional; en el cual, "la organización emplea la comunicación para promover el entendimiento mutuo" (Di Genova, 2012, pág. 13). Dicho de otro modo, esta no se limitaría más a emitir mensajes con la sola intención de informar o influir 
positivamente en la opinión pública; sino que buscaría predisponer a sus públicos favorablemente hacia sí misma mediante el descubrimiento de los deseos y expectativas de sus receptores, y adecuando su intencionalidad a tales hallazgos.

Pese a los avances de la disciplina, y la evidente necesidad de gestionar la relación entre la organización y sus públicos estableciendo una relación bidireccional que permita la retroalimentación constante; las funciones que ejerce la Jefatura de Prensa y RRPP continúan siendo meramente informativas, lo cual no satisface las necesidades comerciales ni institucionales de la organización. La miopía empresarial de los dirigentes 'santos' y/o el desinterés por obtener réditos económicos a través del fútbol son las causas del manejo del club, cuyos hinchas son sumamente escasos a pesar de ostentar dos títulos en su palmarés. De no ser por la subvención económica de la Universidad San Martín de Porres, el club de fútbol subsistiría apenas jugando en la liga distrital de Santa Anita, y no en la primera división.

En contraste, el comité de comunicación del Club Sporting Cristal asume competencias que trascienden la sola relación con los medios de prensa. De hecho, los profesionales responsables desempeñan una serie de acciones de comunicación orientadas a reforzar la identidad del club, dotándola de valores diferenciales que se engloban en la definición de la 'Raza Celeste'. De esa manera, pretenden alinear la conducta de los integrantes de la organización, principalmente la de los jugadores de todas las categorías tanto dentro como fuera de la cancha. Ello con la finalidad de aminorar cualquier daño a la imagen del club e incrementar el interés de la empresa privada por invertir patrocinando al primer equipo.

La identidad es el activo principal de toda entidad deportiva, ya que en ella radica la posibilidad de establecer profundos vínculos emocionales con los aficionados (Sanahuja Peris, 2012, pág. 175). En la medida que el club logre transmitir de manera eficiente sus atributos de identidad a los públicos a través de una comunicación y experiencia de marca coherente y coordinada, será capaz de congregar gran número de fans sin depender de los resultados deportivos. Además, le permitirá construir una imagen de marca relevante y atractiva, no sólo para los fans, sino también para las empresas interesadas en asociar su marca al club (Varela \& Franch, 2014, pág. 14). 
Y es que, como bien señala Costa (2010, pág. 96), no se trata de 'vender' una identidad para crear en el mercado una imagen delineada por el jefe de publicidad o de relaciones públicas; sino, de comunicar conceptos de la misma mediante vivencias, experiencias y mensajes acordes que contribuyan a crear una imagen global. Evidentemente, ésta será global en la medida en la que logre integrar la cultura y la comunicación interna y externa.

Ahora bien, lamentablemente para los intereses comerciales de ambas instituciones, se percibe - más en el Club Deportivo San Martín Porres (USMP) que en el Club Sporting Cristal (SC) - un conocimiento todavía exiguo del negocio en el que se encuentran inmersos. El mismo que, según Soriano (2013, pág. 26), corresponde al suministro de entretenimiento a los aficionados y al público en general, compitiendo con otras actividades de ocio como lo son el cine, el teatro, etc.

Toda entidad deportiva debe concebirse a sí misma como un proveedor de servicios de entretenimiento; y la marca del club, como "la síntesis de la experiencia que supone pertenecer a una comunidad de aficionados con una emoción en común" (Sanahuja Peris \& Blay Arráez, 2013). Esta visión abre la posibilidad de diversificar las fuentes de ingresos y establecer un nuevo tipo de relación con los actores que forman parte del espectáculo deportivo (Gómez, 2014, pág. 37).

La limitada gestión de marketing deportivo en los clubes peruanos impide la explotación de fuentes de ingreso no tradicionales para el fútbol peruano, pero desarrolladas con suma eficiencia en países vecinos. El SC, por ejemplo, restringe sus actividades de marketing deportivo a promociones dirigidas a incrementar la venta de tickets al estadio y oferta de algunos artículos de merchandising. Empero, no existe mejor inversión en marketing que invertir en mejorar el producto; es decir en la experiencia que los fanáticos viven antes, durante y después del partido. Incluso cuando no se juegan partidos del campeonato. Los esfuerzos de SC en ese aspecto todavía son exiguos.

Está claro, los clubes deben recurrir a las técnicas de marketing presencial debido a la experiencia que vende, la cual debe destacar por la emoción de la atmósfera que abruma el deporte. Sin embargo, ¿Qué suele ocurrir en nuestro país? Lamentablemente para quienes disfrutamos del fútbol, acudir a un espectáculo deportivo en nuestro país no es una actividad placentera. Nada más que la remota 
posibilidad de gritar un gol del equipo con el cual se identifica convence a un aficionado a invertir su tiempo en trasladarse a un recinto deportivo.

Claramente, la profesionalización del fútbol peruano es necesaria en aras del desarrollo de la industria del deporte en nuestro país. Pues bien, dado que todo club deportivo requiere ser concebido como sujeto de comunicación y fuente de identidad a fin de generar valor, la incorporación de la función estratégica de la comunicación corporativa en la gestión de los mismos, resulta fundamental. Así, la comunicación en las entidades deportivas no se verá limitada a funciones informativas, ni el marketing sólo a promover la venta de entradas.

Es decir, como el resto de organizaciones, las entidades deportivas deben considerar la gestión estratégica de los vectores del paradigma del Siglo XXI, propuesto por Costa (2003): La identidad, la cultura, la acción, la comunicación y la imagen. No obstante, superar con efectividad el desafío de monetizar la emoción de los fanáticos dependerá en gran medida del conocimiento de la industria deportiva y las tendencias actuales. Los clubes no deben pasar de ser percibidos como asociaciones barriales a ser gestionadas como verdaderas proveedoras de entretenimiento. ¿Podrá ello devolverle al Perú los éxitos deportivos que tanto anhela la actual generación? No resolverá el problema de resultados, pero podrá aportar muchísimo. 


\section{Referencias}

Agudo, Á., \& Toyos, F. (2003). Marketing del fútbol. Madrid, España: ESIC Editorial.

Campuzano, J. C. (25 de Abril de 2013). Home: Juan Carlos Campuzano S. Recuperado el 10 de Setiembre de 2015, de sitio web de Juan Carlos Campuzano S.:

https://jcampuza.wordpress.com/2013/04/25/fc-barcelona-el-exito-de-unaestrategia-empresarial-y-deportiva/

Costa, J. (2003). Imagen Corporativa en el Siglo XXI. Buenos Aires: La Crujía.

Costa, J. (2009). El DirCom hoy. Barcelona: Costa Punto Com Editor.

Costa, J. (2009). El DirCom hoy. Dirección y gestión de la Comunicación en la nueva economía. . Barcelona: Costa Punto Com Editor.

Costa, J. (2010). El ADN del DirCom. Origen, necesidad, expansión y futuro de la Dirección de Comunicación. Barcelona: Costa Punto Com Editor.

Deloitte. (2010). Football Money League. Sports Business Group.

Di Genova, A. E. (2012). Manual de relaciones públicas e institucionales: estrategias de comunicación y tácticas relacionales. Buenos Aires, Argentina: Ugerman Editor.

Ginesta, X. (2011). El fútbol y el negocio del entretenimiento global. Los clubes como multinacionales del ocio. Comunicación y Sociedad, XXIX(1), 141-166.

Gómez, S. (Junio de 2014). Características estructurales de los clubes de fútbol profesional en España. Harvard Deusto Business Review(235), 34-42.

Gómez, S. (Junio de 2014). Características estrutcurales de los clubs de fútbol profesional en España. Harvard Deusto Business Review(235), 34 - 42.

Kuper, S., \& Szymanski, S. (2009). iEl fútbol es así! (soccernomics). (D. Menezo García, Trad.) New York, Estados Unidos de América: Nation Books.

Lacasa, A. (2004). Gestión de la comunicación empresarial. Barcelona, España: Gestión 2000.

López, H. (2012). El rol de los medios en la transformación del deporte en bien de consumo. Doxa.comunicación(18), 13-28.

Martín, F. (2008). Comunicación Empresarial: necesidad y deber social. Recuperado el Enero de 2016, de Revistas Universidad Complutense de Madrid: http://revistas.ucm.es/index.php/MESO/article/view/22520

Miller, R. M., Murillo, C., \& Seitz, O. (2012). El fútbol como negocio. Una introducción a la gestión deportiva en Europa y Brasil. (M. Monalve Z., Ed.) Lima, Perú: Universidad del Paífico. 
Olabe, F. (2009). La comunicación no convencional en los clubes de fútbol. Pensar la Publicidad, III(1), 121-138.

Olabe, M. (2012). La gestión comunicativa de los clubes de fútbol en España con los medios de comunicación como público estratégico: análisis del modelo de FC Barcelona y del Real Madrid CF y su percepción por los periodistas (Tesis Doctoral). Recuperado el 30 de Setiembre de 2015, de Sidio web de Educación:

https://www.educacion.gob.es/teseo/imprimirFicheroTesis.do?fichero=36792

Rinke, S. (21 de Agosto de 2007). ¿La última pasión verdadera? Historia del fútbol en América Latina en el contexto global. Iberoamericana, 7(27), 85-100. Recuperado el 29 de Mayo de 2015, de http://www.iai.spkberlin.de/fileadmin/dokumentenbibliothek/Iberoamericana/2007/Nr_27/27_Rinke.pd $f$

Rivera, J., \& Morelo, V. (2012). Marketing y fútbol. El mercado de las pasiones. Madrid: Esic Editorial.

Sanahuja Peris, G. (2012). Análisis de los procesos en la comunicación corporativa de los clubes deportivos profesionales españoles. Estudio Exploratorio. adComunica. Revista Científica de Estrategias, Tendencias e Innovación en Comunicación(3), 171-189.

Sanahuja Peris, G., \& Blay Arráez, R. (2013). Retos para alcanzar la excelencia en comunicación corporativa en los clubs deportivos españoles. Revista Mediterránea de Comunicación, $4(1), 53-84$.

Soriano, F. (31 de 07 de 2013). La pelota no entra por azar. Buenos Aires: Granica. Recuperado el 20 de Julio de 2015, de Nuestra marca PwC: Spotlight con Ferrán Soriano: https://www.youtube.com/watch?v=JiT2OwdSAUs

Varela, A., \& Franch, J. (Junio de 2014). La gestión de las marcas en el deporte. Harvard Deusto Business Review(235), 6 - 18.

Vilagut, J. (2011). Mensajes Redondos. Madrid: LID Editorial Empresarial. 